\title{
UTILIZAÇÃO DA DIETA CETOGÊNICA COMO ESTRATÉGIA PARA O MANEJO DE PACIENTES COM EPILEPSIA REFRATÁRIA: UMA REVISÃO DA LITERATURA
}

\author{
Use of the ketogenic diet as a strategy for the management of patients with refractory epilepsy: A \\ literature review
}

Douglas Kovacs dos Santos ${ }^{1}$, Paolo Ruggero Errante ${ }^{2}$, Luis Carlos Barbosa Pontes Junior ${ }^{3}$, Alexandre Raphael Junior ${ }^{4}$, Patrícia Colombo-Souza ${ }^{5}$, Renato Ribeiro Nogueira Ferraz ${ }^{6}$, Francisco Sandro Menezes-Rodrigues ${ }^{7}$.

${ }^{1}$ Farmacêutico. Especialista em Farmacologia Clínica pelas Faculdades Oswaldo Cruz (FOC), São Paulo, SP, Brasil. ORCID: https://orcid.org/0000-0002-5740-3295. E-mail: kovacs.douglas@gmail.com. ${ }^{2}$ Médico Veterinário. Doutor em Imunologia pela Universidade de São Paulo (USP). Pesquisador no Departamento de Farmacologia da Escola Paulista de Medicina - Universidade Federal de São Paulo (EPM-UNIFESP), São Paulo, SP, Brasil. ORCID: https://orcid.org/0000-0002-6997-7587. E-mail: errantepr@yahoo.com. ${ }^{3}$ Graduando em Medicina na Universidade Santo Amaro (UNISA), Santo Amaro, SP, Brasil. ORCID: https://orcid.org/0000-0002-7376-906X. E-mail: engluiscbp@hotmail.com. ${ }^{4}$ Odontologista. Mestre em Análises Clínicas pela Universidade Santo Amaro (UNISA). Professor da Universidade Santo Amaro, São Paulo. ORDCID: https://orcid.org/0000-0003-1697-5067. E-mail: aaraphael@prof.unisa.br. ${ }^{5}$ Nutricionista. Doutora em Nutrição pela Universidade Federal de São Paulo (UNIFESP). Coordenadora de Pesquisa da Universidade Santo Amaro (UNISA). ORCID: https://orcid.org/0000-0003-0247-4245. E-mail: pcolombo@prof.unisa.br. ${ }^{6}$ Biólogo. Doutor em Ciências pela Escola Paulista de Medicina - Universidade Federal de São Paulo (EPM-UNIFESP). Pesquisador Convidado do Programa de Pós-Graduação em Políticas Públicas - Universidade de Mogi das Cruzes (UMC), Mogi das Cruzes, SP, Brasil. ORDCID: https://orcid.org//0000-0002-8931-895X. E-mail: renatobio@hotmail.com. ${ }^{7}$ Farmacêutico-Bioquímico. Doutor em Farmacologia pela Escola Paulista de Medicina - Universidade Federal de São Paulo (EPM-UNIFESP). Pesquisador e Docente das Residências Multiprofissionais da Escola Paulista de Medicina - Universidade Federal de São Paulo (EPM-UNIFESP), São Paulo, SP, Brasil. ORCID: https://orcid.org/0000-0001-7913-0585. E-mail: sandromrodrigues@hotmail.com.

\section{Resumo}

Introdução: A epilepsia é uma doença que afeta milhões de pessoas em todas as partes do mundo. Crises epilépticas se apresentam nos pacientes de forma parcial ou generalizada, dependendo do grau de atividade da doença. Atualmente, há muitos fármacos disponíveis no mercado, os quais possuem diversos mecanismos de ação e que podem ser utilizados de forma isolada ou concomitante. No entanto, em alguns pacientes, principalmente, em crianças e adolescentes, esses fármacos se mostram ineficazes e incapazes de promover o controle tanto da frequência quanto da intensidade das crises epilépticas, a quais são denominadas de epilepsia refratária. Assim, a busca por tratamentos alternativos capazes de controlar as frequência e intensidade das crises epilépticas refratárias é incessante e intensa e, dentre as estratégias 
usadas, atualmente, destaca-se a dieta cetogênica (DC), que consiste numa elevada ingestão de lipídios e baixa ingestão de carboidratos e proteínas e que tem fornecido uma melhora na qualidade de vida de pacientes, principalmente, em crianças e adolescentes com epilepsia refratária. Objetivo: Realizar uma revisão da literatura sobre os benefícios causados pela utilização da DC, como tratamento adjuvante e/ou alternativo, no tratamento da epilepsia refratária, principalmente, em crianças e adolescentes. Metodologia: O estudo foi realizado por meio de uma revisão bibliográfica e integrativa da literatura, através da compilação de artigos científicos e livros sobre o tema proposto. As informações foram pesquisadas na base de dados Google acadêmico, Scielo e PubMed. As palavras-chave utilizadas para a busca dos artigos científicos foram: Epilepsia, Epilepsia refratária, Dieta cetogênica. Para a elaboração deste estudo foram pesquisadas publicações a partir de 2008 a 2018, levando em consideração o fator atualidade e relevância do tema, cujo título estabelecia a relação com o estudo. Das publicações encontradas, 22 foram utilizadas. Conclusão: Conclui-se que a DC se apresenta como uma importante opção terapêutica muito segura, barata e eficaz e que, por isso, pode ser utilizada no manejo de pacientes com epilepsia refratária, principalmente, em crianças e adolescentes.

Palavras-chave Epilepsia Refratária, Dieta Cetogênica, Qualidade de vida.

\begin{abstract}
Introduction: Epilepsy is a disease that affects millions of people in all parts of the world. Epileptic seizures occur in patients in a partial or generalized way, depending on the degree of disease activity. Currently, there are many drugs available in the market, which have several mechanisms of action and can be used alone or concomitantly. However, in some patients, especially in children and adolescents, these drugs are ineffective and incapable of promoting the control of both the frequency and severity of epileptic seizures, which are called refractory epilepsy. Thus, the search for alternative treatments capable of controlling the frequency and intensity of refractory epileptic seizures is incessant and intense, and among the strategies currently used, the ketogenic diet (DC), which consists of a high lipid intake and low carbohydrate and protein intake and has provided an improvement in patients' quality of life, mainly in children and adolescents with refractory epilepsy. Objective: To review the literature on the benefits of $\mathrm{CD}$, as an adjuvant and alternative treatment, in the treatment of refractory epilepsy, mainly in children and adolescents. Methodology: The study was carried out through a bibliographical and integrative review of the literature, through the compilation of scientific articles and books on the proposed theme. The information was searched in the Google academic database, Scielo and PubMed. The keywords used to search the scientific articles were: Epilepsy, Refractory Epilepsy, Ketogenic Diet. For the preparation of this study, publications were searched from 2008 to 2018, taking into account the relevance and relevance of the theme, whose title established the relationship with the study. Of the publications found, 22 were used. Conclusion: It is concluded that CHD presents itself as an important therapeutic option that is very safe, cheap and effective and, therefore, can be used in the management of patients with refractory epilepsy, mainly in children and adolescents.
\end{abstract}

Keywords: Refractory epilepsy, Ketogenic diet, Quality of life.

\title{
Introdução
}

O estado epiléptico é definido como uma condição resultante do fracasso dos mecanismos responsáveis pelo término da convulsão ou a partir do início de mecanismos que levam a convulsões anormalmente prolongadas. É uma condição que pode ter consequências em longo prazo, incluindo morte 
ou lesão neuronal e alteração de redes neuronais, dependendo do tipo e duração das crises. Pode ser classificados de acordo com sua semiologia (presença ou ausência de sinais e sintomas motores proeminentes e do grau de comprometimento da consciência), etiologia, registro do eletroencefalograma e idade do paciente (GOMES et al., 2018).

Mais de dois milhões de casos são diagnosticados com epilepsia a cada ano, em crianças, a taxa de incidência anual de epilepsia é de 5 a 7 por 100.000 e a taxa de prevalência varia de 20 a 60 por 100.000 (KINDEREN et al., 2011; REZAEI et al., 2019). Estima-se que 1 a cada 26 pessoas irão desenvolver epilepsia em algum momento da vida. Na população mundial cerca de $50 \%$ dos casos iniciam-se na infância e adolescência. A cada ano surgem aproximadamente 3 milhões de novos casos no mundo. Nos Estados Unidos, Canadá e União Europeia sua prevalência varia de 3,6 a 6,5 por cada 1000 crianças. Na África e na América Latina essa prevalência é de 6,6 a 17 por 1000 crianças (SILVA et al., 2014).

O diagnóstico de epilepsia é feito através da realização de anamnese, exames neurológicos (eletroencefalograma e ressonância magnética) e exames laboratoriais. $\mathrm{Na}$ maioria dos pacientes, a farmacoterapia gera uma redução dos sinais e sintomas, e proporciona uma maior qualidade de vida de forma transitória (SILVA, CABRAL, 2008). O EEG é um teste complementar decisivo na avaliação diagnóstica, evolução, prognóstico e decisão terapêutica, mas atualmente não há critérios de EEG baseados em evidências para o estado epilético (GOMES et al., 2018).

O tratamento deve ser iniciado durante o atendimento pré-hospitalar (quando possível) e conforme a configuração da sala de emergência. Benzodiazepínicos intermitentes são os fármacos de primeira linha para o tratamento nesta fase. Se as convulsões persistirem, drogas antiepilépticas (DAEs) devem ser iniciadas. A monoterapia é indicada em primeiro lugar, mas em segundo plano, as DAEs devem ser associadas se as convulsões persistirem (KOSE et al., 2018)

Se a atividade epiléptica clínica e/ou eletroencefalográfica persistir, apesar de duas doses apropriadamente selecionadas e drogas antiepilépticas, o paciente é considerado em estado refratário. Diferentes estratégias de tratamento são defendidas, dependendo da forma clínica. Para pacientes com epilepsia refratária, há indicação imediata para anestesia geral e confirmação da remissão completa das crises por eletroencefalografia (EEG) (GOMES et al., 2018). Embora, os medicamentos antiepilépticos continuem sendo a terapia de primeira linha para a epilepsia, quase $30 \%$ dos pacientes não respondem bem aos anticonvulsivantes e se submetem a outros tratamentos, incluindo dieta cetogênica (DC), estimulação do nervo vago e cirurgia (KAYYALI et al., 2016; PREZIOSO et al., 2018)

Vários estudos avaliaram os efeitos potenciais da dieta e suplementos dietéticos como tratamento adjunto nos vários distúrbios neurológicos, particularmente na epilepsia. A DC, uma das dietas mais estudadas na gestão da epilepsia, é uma dieta especial composta por alto teor de gordura, baixo teor de carboidratos e moderada quantidade de proteína (SESSA, FERRAZ, 2019). Foi utilizada pela primeira vez em 1921 para tratar a epilepsia intratável (HEO et al., 2017). A DC e outras terapias dietéticas são geralmente utilizadas em pacientes com epilepsia refratária que não conseguiram alcançar o controle com tratamentos medicamentosos (HEO ET AL., 2017). Resultados promissores em adultos com epilepsia refratária foram relatados recentemente em uma série de casos, sendo eficaz em $90 \%$ dos pacientes como último recurso terapia, em uma media de três dias após a cetose ser estabelecida (GÜZEL et al., 2016).

A dieta cetogênica é uma das terapias mais eficazes para a epilepsia intratável na população adulta e pediátrica. Por outro lado, a DC pode causar efeitos adversos, como crescimento de estatura prejudicado, constipação, deficiência de selênio e cardiomiopatia, pedras nos rins e hiperlipidemia (KOSE et al., 2018). 
Como qualquer outro tratamento, as dietas cetogênicas não estão isentas de riscos e exigem um monitoramento cuidadoso para evitar ou efeitos adversos em longo prazo. No entanto, os efeitos colaterais mais comuns são geralmente transitórios, pequenos e podem ser tratados com a descontinuação da mesma (PREZIOSO et al., 2018). Vários estudos avaliaram a eficácia da DC no tratamento da epilepsia infantil refratária, mostrando dados promissores quanto à eficácia e tolerabilidade. Nesta revisão, concentramonos na eficácia da DC como terapia adjuvante no manejo de paciente com epilepsia refratária, principalmente, em crianças e adolescentes.

\section{Objetivo}

Realizar uma revisão da literatura sobre os benefícios causados pela utilização da DC, como tratamento adjuvante e/ou alternativo, no tratamento da epilepsia refratária, principalmente, em crianças e adolescentes.

\section{Método}

O estudo foi realizado por meio de uma revisão bibliográfica e integrativa da literatura, através da compilação de artigos científicos e livros sobre o tema proposto. As informações foram pesquisadas na base de dados Google acadêmico, Scielo e PubMed. As palavras-chave utilizadas para a busca dos artigos científicos foram: Epilepsia, Epilepsia refratária, Dieta cetogênica. Para a elaboração deste estudo foram pesquisadas publicações a partir de 2008 a 2018, levando em consideração o fator atualidade e relevância do tema, cujo título estabelecia a relação com o estudo. Das publicações encontradas, 22 foram utilizadas.

\section{Revisão da Literatura}

A DC consiste numa dieta com finalidade terapêutica que começou a ser utilizada durante a década de 20, nos Estados Unidos, em pacientes com crises epiléticas graves. No entanto, com o passar dos anos, os novos e eficientes fármacos desenvolvidos, assim como novas formas de tratamento como, tratamentos cirúrgicos, fizeram com que a DC fosse esquecida. Em meados da década de 90, a DC voltou a ser utilizada e indicada para pacientes com epilepsia refratária (ER), principalmente, em crianças e adolescentes (PEREIRA et al., 2009).

A DC deve ser prescrita de forma individual, precisamente calculada e balanceada para a altura e peso ideal da criança e adolescente, sendo que esta dieta consiste em uma alimentação rica em lipídios, moderada em proteínas e pobre em carboidratos (PEREIRA et al., 2009).

No início do tratamento, recomenda-se que o paciente seja internado para a retirada de alimentos sólidos e líquidos por um período mínimo de 36 horas ou até a detecção de aumento de corpos cetônicos no organismo. Neste período, devem-se tomar cuidados especiais com o paciente, que pode apresentar quadros de hipoglicemia e desidratação. Em alguns casos, inicia-se uma pré-dieta na qual a ingestão de carboidratos é diminuída para uma melhor adaptação do paciente à DC (KINDEREN et al., 2011).

Com a ingestão excessiva de lipídios, o paciente apresenta-se num estado inanição. A partir deste momento os lipídios são utilizados como a principal fonte de energia para o organismo, mantendo o corpo num estado de cetose, o qual aumenta a estabilidade neural e os níveis de GABA nos terminais nervosos direta ou indiretamente, principalmente, em pacientes refratários ao tratamento farmacológico (NONINOBORGES et el., 2004).

A DC apresenta um efeito antiepilético e neuroproteção por inibir a apoptose intercambiada pelos neurônios do hipocampo. Como a grande parte dos precursores dos corpos cetônicos é constituída por 
ácidos graxos de cadeia longa que são liberados do tecido adiposo em resposta à diminuição da glicemia sanguínea. Como consequência desse processo, a insulina plasmática se eleva (VEECH, 2004).

Os corpos cetônicos produzidos atravessam a barreira hematoencefálica, sendo utilizados como substrato para o ciclo de Krebs e cadeia respiratória no metabolismo energético cerebral. Os ácidos graxos ao se oxidarem produzem grande quantidade adenosina trifosfato, elevando as reservas energéticas cerebrais, alterando a bioquímica dos neurônios, promovendo inibição da excitabilidade neural exacerbada e, desta forma, induzindo um efeito neuroprotetor (PEREIRA et al., 2009; PREZIOSO et al., 2018).

Como o cérebro das crianças tem uma aderência maior ao metabolismo de corpos cetônicos, explica uma maior eficiência da DC em crianças do que em adultos. Normalmente, a DC é iniciada na proporção de 2:1 (duas partes de lipídios e uma parte de proteínas e carboidratos) com o passar dos dias vai evoluindo até chegar a proporção de $4: 1$ (90\% de lipídios, $8 \%$ proteínas e $2 \%$ de carboidratos ou 4 gramas de lipídios para cada $0,5 \mathrm{~g}$ de proteína mais $0,5 \mathrm{~g}$ carboidrato). Essa proporção varia dependendo da aceitação e tolerância de cada paciente a DC (NAKAHARADA, 2008). Estima-se que a após três ou quatro semanas o metabolismo cerebral já esteja adaptado e os neurônios passam a utilizar corpos cetônicos como principal fonte de energia (PEREIRA et al., 2009; PREZIOSO et al., 2018).

A DC se apresenta de forma efetiva por dois a três anos, podendo variar conforme a resposta esperada. A cada trimestre são realizados exames metabólicos de controle (ácido úrico, dosagem de eletrólitos, perfil lipídico, hemograma, gasometria, entre outros) (VERONIQUE et al., 2015; CERVENKA et al., 2016; REZAEI et al., 2019).

Alguns sintomas podem aparecer por um curto ou longo espaço de tempo. Reações adversas também são comuns e podem ser de curto ou longo prazo, dentre as reações adversas de curto prazo se destacam obstipação, diarreia, vômitos, hipoglicemia, sonolência, letargia entre outras (PRUDENCIO et al., 2017; PREZIOSO et al., 2018).

Reações adversas de longo prazo incluem dislipidemias, cálculo renal, alterações metabólicas recorrentes infecções, acidose metabólica, hiperuricemia, irritabilidade, recusa alimentar. A resistência e a desistência durante o início e continuação do tratamento, são os grandes vilões da ineficácia da DC (PEREIRA et al., 2009).

A DC pode diminuir a produção do fator de crescimento semelhante à insulina 1 (IGF-1), utilizada no fígado em resposta ao hormônio do crescimento. A DC não promove alterações relacionadas ao peso, mantendo as necessidades energéticas estimadas para crianças saudáveis. Porém, crianças em uso terapêutico da DC requerem monitoramento cuidadoso do seu crescimento (GROLEAU et al., 2015). Atualmente tem-se trabalhado para transformar a DC agradável, palatável e de fácil administração, aumentando a aderência dos (SAMPAIO, 2016).

A DC diminui as visitas às salas de emergências hospitalares e a hospitalização de pacientes com crises epilépticas refratárias, mantendo o controle das crises e diminuindo assim os custos no serviço de atendimento e internação (KAYYALI; LUNIOVA; ABDELMOITY, 2016).

No estudo de Heo et al (2017), foi avaliado o efeito da DC e outras terapias dietéticas sobre as concentrações séricas das drogas antiepiléticas em pacientes com epilepsia. Quando a DC e outras terapias dietéticas foram usados em combinação com as dorgas antiepiléticas, apenas as concentrações séricas de ácido valproico diminuiram. Portanto, os níveis séricos de ácido valpróico devem ser cuidadosamente monitorados durante a utilização da DC e outras terapias dietéticas (HEO ET AL., 2017). Kverneland et al. encontraram um aumento considerável nas concentrações séricas de drogas antiepiléticas com o uso 
da dieta do Dr. Atkins, mas Dahlin et al. (2006) não encontraram alterações nas concentrações plasmáticas de clonazepam, lamotrigina, fenobarbital, topiramato e acido valpórico. Em conclusão, a DC e outras terapias dietéticas podem alterar as concentrações séricas de fármacos antiepiléticos, especialmente, do ácido valpróico e, consequentemente, aumentar as chances de intervalos terapêuticos alvo. Assim, o monitoramento rigoroso das concentrações séricas dos fármacos antiepiléticos deve ser considerado quando a DC e outras terapias alimentares forem utilizadas concomitantemente.

Em um estudo longitudinal realizado com pacientes utilizando a dieta do Dr. Atkins para o tratamento de convulsões por mais de 3 meses até 2 anos, não houve relato de eventos cardio ou cerebrovasculares. Em relação ao colesterol total e a lipoproteina de baixa densiade (LDL), inicialmente aumentaram ao longo de 3 meses e normalizaram com o tratamento dietético prolongado (CERVENKA et al., 2016).

Os resultados preliminares de um estudo multicêntrico parecem indicar que a dieta cetogênica pode ser útil no controle das crises em uma porcentagem de crianças que não tiveram suas crises controladas com medicamentos. Aproximadamente 33\% dessas crianças alcançam controle completo e outras 33\% são capazes de melhorar o controle e diminuir seus medicamentos (RAMOS, 2001).

A resposta à dieta é variável. Um número significante de pacientes apresenta diminuição dos episódios de crise, e outros, remissão total. Alguns pacientes não apresentam qualquer resposta ao tratamento. Depois de iniciada a dieta, os resultados podem ser esperados até dois meses, indicando-se interrompê-la após esse período, caso não traga benefício ao paciente (RAMOS, 2001; NONINOBORGES et al., 2004).

A dieta tem se mostrado boa terapêutica nos casos de epilepsia quando as medicações convencionais não controlam as crises adequadamente, ou casos em que os medicamentos causam efeitos adversos que dificultam sua utilização.

\section{Conclusão}

Mediante os dados apresentados, podemos concluir que a DC se apresenta como uma importante opção terapêutica muito segura, barata e eficaz que pode ser utilizada no manejo de pacientes com epilepsia refratária, principalmente, em crianças e adolescentes, uma vez que esses apresentam uma resposta terapêutica mais efetiva nesses pacientes quando comparados aos adultos.

\section{Referências}

DAHLIN, MG; BECK, OML; ÅMARK, PE. Plasma levels of antiepileptic drugs in children on the ketogenic diet. Pediatr Neurol, v. 35, p. 6-10, 2006.

GOMES, D; PIMENTEL, J; BENTES, C; AGUIAR DE SOUZA, D; ANTUNES, AP; ALVAREZ, A; SILVA, ZC. Consensus Protocol for the Treatment of Super-Refractory Status Epilepticus. Acta Med Port, v. 31, n. 10, p. 598-605, 2018.

GÜZEL, O.; YILMAZ, U.; UYSAL, U.; ARSLAN, N. The effect of olive oil-based ketogenic diet on serum lipid levels in epileptic children. Neurol Sci., v. 37, p. 465-470, 2016.

HEO, G.; KIM, S.H.; CHANG, M.J. Effect of ketogenic diet and other dietary therapies on anti-epileptic drug concentrations in patients with epilepsy. J Clin Pharm Ther., v. 42, p. 758-764, 2017.

KAYYALI, H.R.; LUNIOVA, A.; ABDELMOITY, A. Ketogenic Diet Decreases Emergency Room Visits and Hospitalizations Related to Epilepsy. Epilepsy Research and Treatment, p. 1-4, 2016. 
KINDEREN, R.J.A.; LAMBRECHTS， D.A.J.E.; POSTULART, D.; KESSELS， A.G.H.; HENDRIKSEN, J.G.M.; ALBERT, P.; ALDENKAMP, A.P.; EVERS, S.M.A.A.; MAJOIE, M.H.J.M. Research into the (Cost-) effectiveness of the ketogenic diet among children and adolescents with intractable epilepsy: design of a randomized controlled trial. BMC Neurol., v. 11, n. 10, p. 1-10, 2011.

KOSE, E.; GUZEL, O.; ARSLAN, N. Analysis of hematological parameters in patients treated with ketogenic diet due to drug-resistant epilepsy. Neurol Sci., v. 39, p. 85-89, 2018.

KVERNELAND, M.; TAUBOLL, E.; SELMER, K.K.; IVERSEN, P.O.; NAKKEN, K.O. Modified Atkins diet may reduce serum concentrations of antiepileptic drugs. Acta Neurol Scand., v. 131, p. 187190, 2015.

MAGIORKINIS, E.; DIAMANTIS, A.; SIDIROPOULOU, K.; PANTELIADIS, C. Highights in the History of Epilepsy: The Last 200 Years. Epilepsy Research And Treatment, p. 1-13, 2014.

NAKAHARADA, L.M.I. Dieta Cetogênica e Dieta de Atkins Modificada no tratamento da Epilepsia Refratária em Crianças e Adultos. Journal of Epilepsy and Clinical Neurophysiology, v. 2, n. 14, p. 6593, 2008.

NONINO-BORGES, C.B.; BUSTAMANTE, V.C.T.; RABITO, E.I.; INUZUKA, L.M.; SAKAMOTO, A.C.; MARCHINI, J.S. Dieta cetogênica no tratamento de epilepsias farmacorresistentes. Rev. Nutr., v. 17, n. 4, p. 515-521, 2004.

PEREIRA, E.S.; ALVES, M.; SACRAMENTO, T.; ROCHA, V.L. Dieta Cetogênica: Como o uso de uma dieta pode interferir em mecanismos neuropatológicos. Revista de Ciências Médicas e Biológicas, v. 1, $\mathrm{n}$. 9, p. 78-82. 2009

PREZIOSO, G.; CARLONE, G.; ZACCARA, G.; VERROTTI, A. Efficacy of ketogenic diet for infantile spasms: A systematic review. Acta Neurol Scand., v. 137, p. 4-11, 2018.

PRUDENCIO, M.B.; LIMA, P.A.; de FREITAS, M.C.P.; CARTOLANO, F.C.; MURAKAMI, D.K.; DAMASCENO, N.R.T. Ketogenic food pyramid for patients with refractory epilepsy: From theory to clinical practice. Revista de Nutrição, v. 30, n. 1, p. 99-108, 2017.

RAMOS, A.M.F. Eficácia da Dieta Cetogênica no Tratamento da Epilepsia Refratária em Crianças e em Adolescentes. Rev Neurociências, v. 9, n. 3, p. 127-131, 2001.

REZAEI, S.; ABDURAHMAN, A.A.; SAGHAZADEH, A.; BADV, R.S.; MAHMOUDI, M. Short-term and long-term efficacy of classical ketogenic diet and modified Atkins diet in children and adolescents with epilepsy: A systematic review and meta-analysis. Nutritional Neuroscience, v. 22, n. 5, p. 317-334, 2019.

SAMPAIO, L.P.B. Ketogenic diet for epilepsy treatment. Arquivos de Neuro-Psiquiatria, v. 74, n. 10, p. 842-848, 2016.

SESSA, W; FERRAZ, R. R. N. Dieta low carb como estratégia de manejo na remissão do diabetes mellitus insulinorresistente: síntese de evidências. IJHMReview, v. 5, n. 1, 2019.

SILVA, A.V.; CABRAL, F.R. Ictogênese, Epileptogênese e Mecanismo de Ação das Drogas na Profilaxia e Tratamento da Epilepsia. Journal Of Epilepsy And Clinical Neurophysiology, v. 14, n. 2, p. 39-45, 2008. SILVA, C.R.A.; CARDOSO, I.S.Z.O.; MACHADO, N.R. Considerações sobre Epilepsia. Sociedade Pediatria do Rio Grande do Sul: Boletim Cientifico, p. 71-76, 2014.

VEECH, R.L. The therapeutic implications of ketone bodies: the effects of ketone bodies in pathologicalconditions: ketosis, ketogenic diet, redox states, insulin resistance, and mitochondrial metabolism. Prostaglandins Leukot Essent Fatty Acids, v. 70, n. 3, p. 309-19, 2004. 
VERONIQUE, G.; SCHALL, J.I.; STALLINGS, V.A.; BERGQVIST, C.A. Long-term impact of the ketogenic diet on growth and resting energy expenditure in children with intractable epilepsy. National Institutes of Health, v. 56, n. 9, p. 1-15, 2015. 